\title{
Weighted Fractional Differentiation Composition Operators from Mixed-Norm Spaces to Weighted-Type Spaces
}

\author{
D. Borgohain and S. Naik \\ Department of Mathematical Science, Gauhati University, Guwahati 781014, India \\ Correspondence should be addressed to S. Naik; spn20@yahoo.com \\ Received 3 December 2013; Accepted 10 January 2014; Published 27 February 2014 \\ Academic Editor: Frédéric Robert
}

Copyright (c) 2014 D. Borgohain and S. Naik. This is an open access article distributed under the Creative Commons Attribution License, which permits unrestricted use, distribution, and reproduction in any medium, provided the original work is properly cited.

Let $\mathbb{D}$ be an open unit disc in the complex plane $\mathbb{C}$ and let $\varphi: \mathbb{D} \rightarrow \mathbb{D}$ as well as $u: \mathbb{D} \rightarrow \mathbb{C}$ be analytic maps. For an analytic function $f(z)=\sum_{n=0}^{\infty} a_{n} z^{n}$ on $\mathbb{D}$ the weighted fractional differentiation composition operator is defined as $\left(D_{\varphi, u}^{\beta} f\right)(z)=$ $u(z) f^{[\beta]}(\varphi(z))$, where $\beta \geq 0, f^{[\beta]}(z)=\sum_{n=0}^{\infty}(\Gamma(n+1+\beta) / \Gamma(n+1)) a_{n} z^{n}$, and $f^{[0]}(z)=f(z)$. In this paper, we obtain a characterization of boundedness and compactness of weighted fractional differentiation composition operator from mixed-norm space $H(p, q, \phi)$ to weighted-type space $H_{\mu}^{\infty}$.

\section{Introduction}

The classical/Gaussian hypergeometric series is defined by the power series expansion

$$
\begin{aligned}
{ }_{2} F_{1}(a, b ; c ; z) & \equiv F(a, b ; c ; z) \\
& =\sum_{n=0}^{\infty} \frac{(a)_{n}(b)_{n}}{(c)_{n}(1)_{n}} z^{n} \quad(|z|<1) .
\end{aligned}
$$

Here $a, b, c$ are complex numbers such that $c \neq-m, m=0,1$, $2,3, \ldots$, and $(a)_{n}$ is Pochhammer's symbol/shifted factorial defined by Appel's symbol

$$
(a)_{n}:=a(a+1) \cdots(a+n-1)=\frac{\Gamma(a+n)}{\Gamma(a)}, \quad n \in \mathbb{N},
$$

and $(a)_{0}=1$ for $a \neq 0$.

Obviously, $F(a, b ; c ; z) \in H(\mathbb{D})$. Many properties of the hypergeometric series including the Gauss and Euler transformations are found in standard textbooks such as $[1,2]$.

For any two analytic functions $f$ and $g$ represented by their power series expansion,

$$
f(z)=\sum_{n=0}^{\infty} a_{n} z^{n}, \quad g(z)=\sum_{n=0}^{\infty} b_{n} z^{n}
$$

in $|z|<R$, the Hadamard product (or convolution) of $f$ and $g$ denoted by $f * g$ and is defined by

$$
(f * g)(z)=\sum_{n=0}^{\infty} a_{n} b_{n} z^{n}
$$

in $|z|<R^{2}$. Moreover,

$$
\begin{array}{r}
(f * g)(z)=\frac{1}{2 \pi} \int_{|w|=\rho} f(w) g\left(\frac{z}{w}\right) \frac{d w}{w}, \\
|z|<\rho R<R^{2} .
\end{array}
$$

In particular, if $f, g \in H(\mathbb{D})$, we have

$$
\begin{array}{r}
(f * g)(\rho z)=\frac{1}{2 \pi} \int_{0}^{2 \pi} f\left(\rho e^{i t}\right) g\left(z e^{-i t}\right) d t \\
0<\rho<1 .
\end{array}
$$

If $f(z)=\sum_{n=0}^{\infty} a_{n} z^{n} \in H(\mathbb{D})$ and $\beta>0$, then the fractional derivative $f^{[\beta]}$ (see [3]) of order $\beta$ is defined by

$$
f^{[\beta]}(z)=\sum_{n=0}^{\infty} \frac{\Gamma(n+1+\beta)}{\Gamma(n+1)} a_{n} z^{n} .
$$


In terms of convolution, we also have

$$
f^{[\beta]}(z)=\Gamma(1+\beta)[f(z) * F(1,1+\beta ; 1 ; z)] .
$$

For $\beta=0$, we define

$$
f^{[0]}(z)=f(z)
$$

It is obvious to find that the fractional derivative and the ordinary derivative satisfy

$$
f^{[k]}(z)=\frac{d^{k}}{d z^{k}}\left(z^{k} f(z)\right), \quad k=0,1,2, \ldots
$$

Let $u(z) \in H(\mathbb{D})$ be fixed and let $\varphi(z)$ be nonconstant analytic self-map of $\mathbb{D}$. For $\beta \geq 0$ and $f(z)=\sum_{n=0}^{\infty} a_{n} z^{n} \in$ $H(\mathbb{D})$, we can define an operator $D_{\varphi, u}^{\beta}$ on $H(\mathbb{D})$, called a weighted fractional differentiation composition operator, by

$$
D_{\varphi, u}^{\beta}(f(z))=u(z) f^{[\beta]}(\varphi(z)) .
$$

We can regard this operator as a generalization of a multiplication operator and a weighted composition operator. In this paper we study the boundedness and the compactness of weighted fractional differentiation composition operator from mixed-norm spaces to weighted-type spaces. Recall that a positive continuous function $\phi$ on $[0,1)$ is called normal if there is $\delta \in[0,1)$ and $s$ and $t$ with $0<s<t$ such that

$$
\begin{array}{ll}
\frac{\phi(r)}{(1-r)^{s}} \text { is decreasing on }[\delta, 1), & \lim _{r \rightarrow 1} \frac{\phi(r)}{(1-r)^{s}}=0, \\
\frac{\phi(r)}{(1-r)^{t}} \text { is increasing on }[\delta, 1), & \lim _{r \rightarrow 1} \frac{\phi(r)}{(1-r)^{t}}=\infty .
\end{array}
$$

Let $d m(z)=(1 / \pi) r d r d \theta$ be the normalized Lebesgue area measure on $\mathbb{D}$ and let $H(\mathbb{D})$ be the space of all analytic functions on $\mathbb{D}$.

For $0<p, q \leq \infty$, and $\phi$ is normal we denote by $H(p, q, \phi)$ the space of all functions $f \in H(\mathbb{D})$ such that

$$
\|f\|_{H(p, q, \phi)}=\left(\int_{0}^{1} M_{q}^{p}(f, r) \frac{\phi^{p}(r)}{1-r} d r\right)^{1 / p}<\infty,
$$

where $p \in(0, \infty)$ and

$$
\|f\|_{H(\infty, q, \phi)}=\sup _{0<r<1} \phi(r) M_{q}(f, r)<\infty,
$$

where

$$
\begin{gathered}
M_{q}(f, r)=\left(\frac{1}{2 \pi} \int_{0}^{2 \pi}\left|f\left(r e^{i \theta}\right)\right|^{q} d \theta\right)^{1 / q} \text { if } q \in(0, \infty), \\
M_{\infty}(f, r)=\sup _{\theta \in[0,2 \pi]}\left|f\left(r e^{i \theta}\right)\right| .
\end{gathered}
$$

When $p=q$ and $\phi(r)=(1-r)^{(\alpha+1) / p}, \alpha>-1$, then $H(p, q, \phi) \equiv A_{\alpha}^{p}(\mathbb{D})$ (classical weighted Bergman space), defined by

$$
\begin{aligned}
A_{\alpha}^{p}=\{f & \in H(\mathbb{D}):\|f\|_{A_{\alpha}^{p}}^{p} \\
& \left.=(\alpha+1) \int_{\mathbb{D}}|f(z)|^{p}\left(1-|z|^{2}\right)^{\alpha} d m(z)<\infty\right\} .
\end{aligned}
$$

Further, when $\alpha \rightarrow-1+0$ then the natural limit to the weighted Bergman space is the Hardy space $H^{p}$; that is, $A_{-1}^{p} \equiv$ $H^{p}$. For more details on Hardy space, see [4].

Suppose $\mu: \mathbb{D} \rightarrow \mathbb{C}$ is normal and radial; that is, $\mu(z)=$ $\mu(|z|)$. The weighted-type space $H_{\mu}^{\infty}$ consists of all $f \in H(\mathbb{D})$ such that

$$
\|f\|_{H_{\mu}^{\infty}}=\sup _{z \in \mathbb{D}} \mu(z)|f(z)|<\infty
$$

A little version of $H_{\mu}^{\infty}$ is denoted by $H_{\mu, 0}^{\infty}$ as the subset of $H_{\mu}^{\infty}$ consisting of all $f \in H(\mathbb{D})$ such that

$$
\lim _{|z| \rightarrow 1} \mu(z)|f(z)|=0
$$

Note that $H_{\mu, 0}^{\infty}$ is a closed subspace of $H_{\mu}^{\infty}$. For the space $H_{\mu}^{\infty}$, see [5].

For $\mu \equiv 1$, we have the space of bounded analytic functions $H^{\infty}$, where

$$
\|f\|_{H^{\infty}}=\sup _{z \in \mathbb{D}}|f(z)|<\infty .
$$

For $\beta=0, D_{\varphi, u}^{\beta}$ equals the weighted composition operator defined by $\left(u C_{\varphi}\right)(f)(z)=u(z) f(\varphi(z)), z \in \mathbb{D}$, which reduces to the composition operator $C_{\varphi}$ for $u(z) \equiv 1$. During the last century, composition operators were studied between different spaces of analytic functions. There are many papers about these operators. Among other things, they deal with boundedness and compactness of these operators. But we will discuss mostly the mixed-norm spaces. For some recent papers on weighted composition operators on some $H^{\infty}$-type spaces, see [5-14].

If $\beta=1$, we get the operator $D_{\varphi, u}^{1}=M_{u} C_{\varphi}+M_{u \varphi} C_{\varphi} D$ which for $u(z) \equiv 1$ gives $D_{\varphi, 1}^{1}=C_{\varphi}+\varphi C_{\varphi} D$ which for $\varphi(z)=z$ gives $D_{z, u}^{1}=M_{u}+z M_{u} D$ and $u(z)=\varphi^{\prime}(z)$ gives $D_{\varphi, \varphi^{\prime}}^{1}=M_{\varphi^{\prime}} C_{\varphi}+\varphi D C_{\varphi}$. For particular choices of $\beta, u$, and $\varphi$, we obtain many operators which are product, addition, and composition of multiplication and differentiation operators. For more details of these types of operators see [15-21].

Recall that, in [22], Stević characterized the boundedness and compactness of weighted differentiation composition operators from mixed-norm spaces to the weighted-type spaces. Our results can be viewed as generalizations of their results.

Throughout this paper, $C$ denotes a positive constant which may vary for different lines. The notation $A \asymp B$ means there is a positive constant $C$ such that $B / C \leq A \leq C B$. 


\section{Some Lemmas}

We collect some basic lemmas which are useful in the proof of the main results.

Lemma 1 (see [23, Lemma 6]). For $\gamma>-1$ and $m>1+\gamma$ one has

$$
\int_{0}^{1}(1-\rho r)^{-m}(1-r)^{\gamma} \leq c(1-\rho)^{1+\gamma-m}, \quad 0<\rho<1
$$

Lemma 2 (see [24, Proposition 1.4.10]). For $\gamma>1$ one has

$$
\int_{0}^{2 \pi} \frac{1}{|1-z|^{\gamma}} \leq C \frac{1}{(1-|z|)^{\gamma-1}}
$$

Lemma 3 (see [22, Lemma 1]). Assume $0<p, q \leq \infty$, $\phi$ is normal, and $f \in H(p, q, \phi)$. Then, for every $n \in N_{0}$, there is a positive constant $C$ independent of $f$ such that

$$
\left|f^{(n)}(z)\right| \leq C \frac{\|f\|_{H(p, q, \phi)}}{\phi(|z|)\left(1-|z|^{2}\right)^{(1 / q)+n}}, \quad z \in \mathbb{D} .
$$

\section{Main Results}

In the following results we show boundedness of the operator $D_{\varphi, u}^{\beta}$ from mixed-norm space $H(p, q, \phi)$ to weighted-type space $H_{\mu}^{\infty}$. As a consequence we obtain many new results in the form of corollaries.

Lemma 3 is true for the $n$th order derivative of $f \in$ $H(p, q, \phi)$. Here we will give a more general result which involves fractional derivative $f^{[\beta]}$ of $f \in H(p, q, \phi)$.

Proposition 4. Assume $0<p \leq \infty, 1 \leq q \leq \infty$, $\phi$ is normal, and $f \in H(p, q, \phi)$. Then, for every $\beta \geq 0$, there is a positive constant $C$ independent of $f$ such that

$$
\left|f^{[\beta]}(z)\right| \leq C \frac{\|f\|_{H(p, q, \phi)}}{\left(1-|z|^{2}\right)^{(1 / q)+\beta} \phi(|z|)} \quad \forall z \in \mathbb{D} .
$$

Theorem 5. For $\beta \geq 0,0<p \leq \infty, 1 \leq q \leq \infty, u \in H(\mathbb{D})$, $\phi$ and $\mu$ are normal, and $\varphi$ is an analytic self-map of $\mathbb{D}$. Then $D_{\varphi, u}^{\beta}: H(p, q, \phi) \rightarrow H_{\mu}^{\infty}$ is bounded if and only if

$$
M=\sup _{z \in \mathbb{D}} \frac{\mu(z)|u(z)|}{\phi(|\varphi(z)|)\left(1-|\varphi(z)|^{2}\right)^{(1 / q)+\beta}}<\infty .
$$

Moreover, if $D_{\varphi, u}^{\beta}: H(p, q, \phi) \rightarrow H_{\mu}^{\infty}$ is bounded, then the following asymptotic relation holds:

$$
\left\|D_{\varphi, u}^{\beta}\right\|_{H(p, q, \phi) \rightarrow H_{\mu}^{\infty}} \asymp M
$$

Proof of Proposition 4. Let $\beta>0$.
Using integral representation of convolution we have, for $1 \leq q<\infty$,

$$
\begin{aligned}
M_{q}^{q}\left(f^{[\beta]}, \rho r\right) & \frac{1}{2 \pi} \int_{0}^{2 \pi}\left|f^{[\beta]}\left(\rho r e^{i \theta}\right)\right|^{q} d \theta \\
= & \frac{\Gamma(1+\beta)^{q}}{2 \pi} \\
& \times \int_{0}^{2 \pi}\left|(f * F(1,1+\beta ; 1))\left(\rho r e^{i \theta}\right)\right|^{q} d \theta, \\
M_{q}\left(f^{[\beta]}, \rho r\right) & \\
= & \frac{\Gamma(1+\beta)}{2 \pi^{1 / q}} \\
& \times\left[\int_{0}^{2 \pi} \mid \frac{1}{2 \pi} \int_{0}^{2 \pi} f\left(r e^{i \theta} e^{-i t}\right)\right. \\
& \\
= & \left.\times\left. F\left(1,1+\beta ; 1 ; \rho e^{i t}\right) d t\right|^{q} d \theta\right]^{1 / q} .
\end{aligned} .
$$

Since $1 \leq q<\infty$, Minkowski’s inequality gives

$$
\begin{aligned}
& M_{q}\left(f^{[\beta]}, \rho r\right) \\
& \leq \frac{\Gamma(1+\beta)}{2 \pi^{1 / q}} \\
& \times\left[\int _ { 0 } ^ { 2 \pi } \frac { 1 } { 2 \pi } \left(\int_{0}^{2 \pi} \mid f\left(r e^{i(\theta-t)}\right)\right.\right. \\
& \left.=C\left[\frac{1}{2 \pi}\left(\int_{0}^{2 \pi}\left|f\left(r e^{i \theta}\right)\right|^{q} d \theta\right)^{1 / q} \times\left. F\left(1,1+\beta ; 1 ; \rho e^{i t}\right)\right|^{q} d \theta\right)^{1 / q} d t\right] \\
& \left.\quad \times \int_{0}^{2 \pi}\left|F\left(1,1+\beta ; 1 ; \rho e^{i t}\right)\right| d t\right] \\
& =C M_{q}(f, r) M_{1}(F, \rho),
\end{aligned}
$$

where $F(z) \equiv F(1,1+\beta ; 1 ; z)$.

For $q=\infty$, we have

$$
\begin{aligned}
M_{\infty}\left(f^{[\beta]}, \rho r\right) & =\sup _{\theta \in[0,2 \pi]}\left|f^{[\beta]}\left(\operatorname{pre}^{i \theta}\right)\right| \\
& =\sup _{\theta \in[0,2 \pi]}\left|(f * F(1,1+\beta ; 1))\left(\operatorname{pre}^{i \theta}\right)\right|
\end{aligned}
$$




$$
\begin{aligned}
& =\sup _{\theta \in[0,2 \pi]} \mid \frac{1}{2 \pi} \int_{0}^{2 \pi} f\left(r e^{i \theta} e^{-i t}\right) \\
& \quad \times F\left(1,1+\beta ; 1 ; \rho e^{i t}\right) d t \mid \\
& \leq M_{\infty}(f, r) M_{1}(F, \rho) .
\end{aligned}
$$

Putting $\rho=r$, we have, for any $q \in[1, \infty]$,

$$
M_{q}\left(f^{[\beta]}, r^{2}\right) \leq C M_{q}(f, r) M_{1}(F, r) .
$$

Since

$$
\begin{aligned}
M_{1}(F, r) & =\frac{1}{2 \pi} \int_{0}^{2 \pi}\left|F\left(1,1+\beta ; 1 ; r e^{i t}\right)\right| d t \\
& =\frac{1}{2 \pi} \int_{0}^{2 \pi} \frac{1}{\left|1-r e^{i t}\right|^{\beta+1}} d t \\
& \leq \frac{C}{2 \pi(1-r)^{\beta}} \quad(\text { by Lemma } 2),
\end{aligned}
$$

therefore

$$
\begin{aligned}
& M_{q}\left(f^{[\beta]}, r^{2}\right) \leq C M_{q}(f, r) \frac{1}{(1-r)^{\beta}} \\
& (1-r)^{\beta} M_{q}\left(f^{[\beta]}, r^{2}\right) \leq C M_{q}(f, r) .
\end{aligned}
$$

For $0<p<\infty$

$$
\begin{aligned}
\|f\|_{H(p, q, \phi)}^{p}= & \int_{0}^{1} M_{q}^{p}(f, r) \frac{\phi^{p}(r)}{1-r} d r \\
\geq & \int_{0}^{1} M_{q}^{p}\left(f^{[\beta]}, r^{2}\right)(1-r)^{p \beta} \\
& \quad \times \frac{\phi^{p}(r)}{1-r} d r \quad(\text { by }(31)) \\
= & \int_{0}^{1} M_{q}^{p}\left(f^{[\beta]}, r^{2}\right) \frac{\left(1-r^{2}\right)^{p \beta}}{(1+r)^{p \beta}} \frac{\phi^{p}(r)}{1-r} d r \\
\geq & C \int_{0}^{1} M_{q}^{p}\left(f^{[\beta]}, r^{2}\right)\left(1-r^{2}\right)^{p \beta} \frac{\phi^{p}(r)}{1-r} d r .
\end{aligned}
$$

Putting $r^{2}=u$, we have

$$
\begin{aligned}
\|f\|_{H(p, q, \phi)}^{p} \geq C \int_{0}^{1} & M_{q}^{p}\left(f^{[\beta]}, u\right)(1-u)^{p \beta} \\
& \times \frac{\phi^{p}(\sqrt{u})}{1-\sqrt{u}} \cdot \frac{1}{\sqrt{u}} d u \\
\geq C \int_{(1+|z|) / 2}^{(3+|z|) / 4} M_{q}^{p}\left(f^{[\beta]}, u\right)(1-u)^{p \beta} & \\
& \times \frac{\phi^{p}(\sqrt{u})}{1-\sqrt{u}} \cdot \frac{1}{\sqrt{u}} d u
\end{aligned}
$$

$$
\begin{aligned}
\geq & C M_{q}^{p}\left(f^{[\beta]}, \frac{1+|z|}{2}\right) \\
& \times \int_{(1+|z|) / 2}^{(3+|z|) / 4}(1-u)^{p \beta} \frac{\phi^{p}(\sqrt{u})}{1-\sqrt{u}} \cdot \frac{1}{\sqrt{u}} d u \\
\geq & C M_{q}^{p}\left(f^{[\beta]}, \frac{1+|z|}{2}\right) \\
& \times \int_{(1+|z|) / 2}^{(3+|z|) / 4}(1-u)^{p \beta} \frac{\phi^{p}(\sqrt{u})}{1-\sqrt{u}} d u \\
= & C M_{q}^{p}\left(f^{[\beta]}, \frac{1+|z|}{2}\right) \\
& \times \int_{(1+|z|) / 2}^{(3+|z|) / 4}(1-u)^{p \beta} \frac{\phi^{p}(\sqrt{u})}{1-u}(1+\sqrt{u}) d u \\
\geq & C M_{q}^{p}\left(f^{[\beta]}, \frac{1+|z|}{2}\right) \\
& \times \int_{(1+|z|) / 2}^{(3+|z|) / 4}(1-u)^{p \beta} \frac{\phi^{p}(\sqrt{u})}{1-u} d u .
\end{aligned}
$$

By using the following asymptotic relations:

$$
\begin{gathered}
\phi(|z|) \asymp \phi(|w|), \quad w \in B\left(z, \sqrt{\frac{3+|z|}{4}}-|z|\right), \\
1-r=1-|z|, \quad r \in\left[\frac{1+|z|}{2}, \frac{3+|z|}{4}\right],
\end{gathered}
$$

we have

$$
\begin{aligned}
\|f\|_{H(p, q, \phi)}^{p} \geq & C M_{q}^{p}\left(f^{[\beta]}, \frac{1+|z|}{2}\right) \\
& \times \int_{(1+|z|) / 2}^{(3+|z|) / 4}(1-|z|)^{p \beta} \frac{\phi^{p}(|z|)}{1-|z|} d u \\
\geq & C M_{q}^{p}\left(f^{[\beta]}, \frac{1+|z|}{2}\right)(1-|z|)^{p \beta-1} \phi^{p}(|z|) \\
& \times \int_{(1+|z|) / 2}^{(3+|z|) / 4} d u \\
= & C M_{q}^{p}\left(f^{[\beta]}, \frac{1+|z|}{2}\right)(1-|z|)^{p \beta} \phi^{p}(|z|) .
\end{aligned}
$$

A simple calculation using Cauchy's integral formula gives

$$
M_{q}^{p}\left(f^{[\beta]}, \frac{1+|z|}{2}\right) \geq C\left|f^{[\beta]}(z)\right|^{p}(1-|z|)^{p / q} .
$$

The above inequality can be written as the following:

$$
\begin{gathered}
\|f\|_{H(p, q, \phi)}^{p} \geq C\left|f^{[\beta]}(z)\right|^{p}(1-|z|)^{p / q}(1-|z|)^{p \beta} \phi^{p}(|z|), \\
\|f\|_{H(p, q, \phi)} \geq C\left|f^{[\beta]}(z)\right|(1-|z|)^{1 / q}(1-|z|)^{\beta} \phi(|z|),
\end{gathered}
$$




$$
\begin{gathered}
\left|f^{[\beta]}(z)\right| \leq C \frac{\|f\|_{H(p, q, \phi)}}{(1-|z|)^{\beta+(1 / q)} \phi(|z|)}, \\
\left|f^{[\beta]}(z)\right| \leq C \frac{\|f\|_{H(p, q, \phi)}}{\left(1-|z|^{2}\right)^{\beta+(1 / q)} \phi(|z|)} .
\end{gathered}
$$

When $p=\infty$, from inequality (31) we have

$$
\begin{aligned}
&\|f\|_{H(\infty, q, \phi)}= \sup _{0<r<1} \phi(r) M_{q}(f, r) \\
& \geq C \sup _{0<r<1} \phi(r)(1-r)^{\beta} M_{q}\left(f^{[\beta]}, r^{2}\right) \\
& \geq C M_{q}\left(f^{[\beta]}, \frac{1+|z|}{2}\right) \\
& \times \phi(|z|)(1-|z|)^{\beta} \quad(\text { where, } 0<|z|<1) \\
& \geq C\left|f^{[\beta]}(z)\right|(1-|z|)^{1 / q} \phi(|z|)(1-|z|)^{\beta} \\
&\left|f^{[\beta]}(z)\right| \leq C \frac{\|f\|_{H(\infty, q, \phi)}}{(1-|z|)^{(1 / q)+\beta} \phi(|z|)} \\
&\left|f^{[\beta]}(z)\right| \leq C \frac{\|f\|_{H(\infty, q, \phi)}}{\left(1-|z|^{2}\right)^{(1 / q)+\beta} \phi(|z|)} .
\end{aligned}
$$

For $\beta=0$ we have $f^{[\beta]}=f$ and so $M_{q}\left(f^{[\beta]}\right)=M_{q}(f)$. Therefore the proof is similar to the case, $\beta>0$.

Proof of Theorem 5. Suppose (24) holds. We have

$$
\begin{aligned}
\left\|\left(D_{\varphi, u}^{\beta} f\right)(z)\right\|_{H_{\mu}^{\infty}}= & \sup _{z \in \mathbb{D}} \mu(z)\left|\left(D_{\varphi, u}^{\beta} f\right)(z)\right| \\
= & \sup _{z \in \mathbb{D}} \mu(z)|u(z)|\left|f^{[\beta]}(\varphi(z))\right| \\
\leq & \operatorname{Csup}_{z \in \mathbb{D}} \frac{\mu(z)|u(z)|}{\phi(\varphi(z))\left(1-|\varphi(z)|^{2}\right)^{(1 / q)+\beta}} \\
& \times\|f\|_{H(p, q, \phi)} \quad(\text { By Proposition 4) } \\
\leq & C M\|f\|_{H(p, q, \phi)} .
\end{aligned}
$$

Therefore,

$$
\left\|D_{\varphi, u}^{\beta}\right\|_{H(p, q, \phi) \rightarrow H_{\mu}^{\infty}} \leq C M .
$$

Conversely, assume that $D_{\varphi, u}^{\beta}: H(p, q, \phi) \rightarrow H_{\mu}^{\infty}$ is bounded. For a fixed $w \in \mathbb{D}$, let

$$
f_{w}(z)=\frac{\left(1-|w|^{2}\right)^{t+1} F((1 / q)+\beta+t+1,1 ; 1+\beta ; \bar{w} z)}{\phi(|w|)},
$$

where the constant $t$ is from the definition of the normality of the function $\phi$ :

$$
\begin{aligned}
M_{q}^{q}\left(f_{w}, r\right) & \\
= & \frac{1}{2 \pi} \int_{0}^{2 \pi}\left|f_{w}\left(r e^{i \theta}\right)\right|^{q} d \theta \\
= & \frac{\left(1-|w|^{2}\right)^{(t+1) q}}{\phi^{q}(|w|)} \cdot \frac{1}{2 \pi} \\
& \times \int_{0}^{2 \pi}\left|F\left(\frac{1}{q}+\beta+t+1,1 ; 1+\beta ; \bar{w} r e^{i \theta}\right)\right|^{q} d \theta .
\end{aligned}
$$

In view of the well-known Gauss identity [1], of hypergeometric function

$$
F(a, b ; c ; z)=(1-z)^{c-a-b} F(c-a, c-b ; c ; z),
$$

we can rewrite the previous equation to obtain the following:

$$
\begin{aligned}
& M_{q}^{q}\left(f_{w}, r\right) \\
& =\frac{\left(1-|w|^{2}\right)^{(t+1) q}}{\phi^{q}(|w|)} \cdot \frac{1}{2 \pi} \\
& \quad \times \int_{0}^{2 \pi} \mid\left(1-\bar{w} r e^{i \theta}\right)^{((-1 / q)-t-1)} \\
& \quad \times\left. F\left(-\frac{1}{q}-t, \beta ; 1+\beta ; \bar{w} r e^{i \theta}\right)\right|^{q} d \theta .
\end{aligned}
$$

We know the hypergeometric function $F(a, b ; c ; z)$ is bounded if $c-a-b>0$ on $|z| \leq 1$ (see [1]). The hypergeometric function $F\left((-1 / q)-t, \beta ; 1+\beta ; \bar{w} r e^{i \theta}\right)$ is bounded on $|z| \leq 1$ since $1+\beta-((-1 / q)-t)-\beta=1+(1 / q)+t>0$; therefore, using Lemma 2 gives

$$
\begin{aligned}
M_{q}^{q}\left(f_{w}, r\right) \leq & C \frac{\left(1-|w|^{2}\right)^{(t+1) q}}{\phi^{q}(|w|)} \\
& \cdot \frac{1}{2 \pi} \int_{0}^{2 \pi}\left|\left(1-\bar{w} r e^{i \theta}\right)^{((-1 / q)-t-1)}\right|^{q} d \theta \\
\leq & C \frac{\left(1-|w|^{2}\right)^{(t+1) q}}{\phi^{q}(|w|)} \cdot(1-|w| r)^{(-t q-q)}, \\
M_{q}\left(f_{w}, r\right) \leq & C \frac{\left(1-|w|^{2}\right)^{t+1}}{\phi(|w|)} \cdot \frac{1}{(1-|w| r)^{t+1}}
\end{aligned}
$$


Since $\phi$ is normal and using Lemma 1, we get $\sup _{w \in \mathbb{D}}\left\|f_{w}\right\|_{H(p, q, \phi)}<\infty$. Now

$$
\begin{aligned}
& f_{w}^{[\beta]}(z)=\Gamma(1+\beta)\left[f_{w} * F(1,1+\beta ; 1 ; z)\right] \\
& =\Gamma(1+\beta)\left[\frac{\left(1-|w|^{2}\right)^{(t+1)}}{\phi(|w|)}\right. \\
& \times F\left(\frac{1}{q}+\beta+t+1,1 ; 1+\beta ; \bar{w} z\right) \\
& * F(1,1+\beta ; 1 ; z)] \\
& =\Gamma(1+\beta) \frac{\left(1-|w|^{2}\right)^{(t+1)}}{\phi(|w|)} \\
& \times\left[\sum_{n=0}^{\infty} \frac{((1 / q)+\beta+t+1)_{n}(1)_{n}}{(1+\beta)_{n}(1)_{n}}(\bar{w} z)^{n}\right. \\
& \left.* \sum_{n=0}^{\infty} \frac{(1+\beta)_{n}}{(1)_{n}} z^{n}\right] \\
& =\frac{\Gamma(1+\beta)\left(1-|w|^{2}\right)^{(t+1)}}{\phi(|w|)} \\
& \times\left[\sum_{n=0}^{\infty} \frac{((1 / q)+\beta+t+1)_{n}}{(1)_{n}}(\bar{w} z)^{n}\right] \\
& =\frac{\Gamma(1+\beta)\left(1-|w|^{2}\right)^{(t+1)}}{\phi(|w|)(1-\bar{w} z)^{((1 / q)+\beta+t+1)}} .
\end{aligned}
$$

For every $\lambda \in \mathbb{D}$, we have

$$
\begin{aligned}
& C\left\|D_{\varphi, u}^{\beta}\right\|_{H(p, q, \phi) \rightarrow H_{\mu}^{\infty}} \\
& \quad \geq\left\|D_{\varphi, u}^{[\beta]} f_{\varphi(\lambda)}\right\|_{H_{\mu}^{\infty}} \\
& \quad=\sup _{\lambda \in \mathbb{D}} \frac{\mu(\lambda)|u(\lambda)|(1+\beta)}{\phi(|\varphi(\lambda)|)\left(1-|\varphi(\lambda)|^{2}\right)^{(1 / q)+\beta}}, \\
& C\left\|D_{\varphi, u}^{\beta}\right\|_{H(p, q, \phi) \rightarrow H_{\mu}^{\infty}} \\
& \quad \geq \sup _{\lambda \in \mathbb{D}} \frac{\mu(\lambda)|u(\lambda)|}{\phi(\lambda) \mid)\left(1-|\varphi(\lambda)|^{2}\right)^{(1 / q)+\beta}} .
\end{aligned}
$$

Therefore,

$$
\left\|D_{\varphi, u}^{\beta}\right\|_{H(p, q, \phi) \rightarrow H_{\mu}^{\infty}} \geq C M
$$

From (40) and (48) we get the asymptotic relation (25).
If we take $\mu(z) \equiv 1$ in the proof of Theorem 5 , we get the following result.

Theorem 6. For $\beta \geq 0,0<p \leq \infty, 1 \leq q \leq \infty, u \in H(\mathbb{D})$, $\phi$ and $\mu$ are normal, and $\varphi$ is an analytic self-map of $\mathbb{D}$. Then $D_{\varphi, u}^{\beta}: H(p, q, \phi) \rightarrow H^{\infty}$ is bounded if and only if

$$
M=\sup _{z \in \mathbb{D}} \frac{|u(z)|}{\phi(|\varphi(z)|)\left(1-|\varphi(z)|^{2}\right)^{(1 / q)+\beta}}<\infty .
$$

Moreover, if $D_{\varphi, u}^{\beta}: H(p, q, \phi) \rightarrow H^{\infty}$ is bounded, then the following asymptotic relation holds:

$$
\left\|D_{\varphi, u}^{\beta}\right\|_{H(p, q, \phi) \rightarrow H^{\infty}} \asymp M
$$

Theorem 7. For $\beta \geq 0,0<p \leq \infty, 1 \leq q \leq \infty, u \in H(\mathbb{D})$, $\phi$ and $\mu$ are normal, and $\varphi$ is an analytic self-map of $\mathbb{D}$. Then $D_{\varphi, u}^{\beta}: H(p, q, \phi) \rightarrow H_{\mu, 0}^{\infty}$ is bounded if and only if $D_{\varphi, u}^{\beta}:$ $H(p, q, \phi) \rightarrow H_{\mu}^{\infty}$ is bounded and $u \in H_{\mu, 0}^{\infty}$.

Proof. Suppose $D_{\varphi, u}^{\beta}: H(p, q, \phi) \rightarrow H_{\mu, 0}^{\infty}$ is bounded. Then it is clear that $D_{\varphi, u}^{\beta}: H(p, q, \phi) \rightarrow H_{\mu}^{\infty}$ is also bounded, since

$$
\begin{array}{r}
\|u\|_{H_{\mu}^{\infty}}=\left\|D_{\varphi, u}^{\beta} h\right\|_{H_{\mu}^{\infty}} \leq\left\|D_{\varphi, u}^{\beta} h\right\|_{H(p, q, \phi) \rightarrow H_{\mu, 0}^{\infty}}\|h\|_{H(p, q, \phi)} \\
\text { where } h(z) \equiv 1 \forall z \in \mathbb{D} .
\end{array}
$$

Therefore, $u \in H_{\mu, 0}^{\infty}$.

Conversely, assume that $D_{\varphi, u}^{\beta}: H(p, q, \phi) \rightarrow H_{\mu}^{\infty}$ is bounded and $u \in H_{\mu, 0}^{\infty}$. Let $p$ be any polynomial. Then,

$$
\begin{aligned}
\mu(z)\left|\left(D_{\varphi, u}^{\beta} p\right)(z)\right| & =\mu(z)|u(z)|\left|p^{[\beta]}(\varphi(z))\right| \\
& \leq \mu(z)|u(z)|\left\|p^{[\beta]}\right\|_{\infty} .
\end{aligned}
$$

Let $p(z)=\sum_{n=0}^{k} a_{n} z^{n}$, where $k<\infty$. Consider the following:

$$
\begin{aligned}
p^{[\beta]}(z) & =\Gamma(1+\beta)[p(z) * F(1,1+\beta ; 1 ; z)] \\
& =\Gamma(1+\beta)\left[\sum_{n=0}^{k} a_{n} z^{n} * \sum_{n=0}^{\infty} \frac{(1+\beta)_{n}}{(1)_{n}} ; z^{n}\right] \\
& =\Gamma(1+\beta)\left[\sum_{n=0}^{k} \frac{(1+\beta)_{n}}{(1)_{n}} a_{n} z^{n}\right] .
\end{aligned}
$$

Obviously, $p^{[\beta]}(z)$ is bounded in $|z|<1$. Also, $u \in H_{\mu, 0}^{\infty}$; therefore $p^{[\beta]} \in H_{\mu, 0}^{\infty}$. Since the set of polynomials is dense in $H(p, q, \phi)$, therefore we have from the proof of Theorem 7 of [22] that

$$
D_{\varphi, u}^{\beta}(H(p, q, \phi)) \subseteq H_{\mu, 0}^{\infty}
$$

from which the boundedness of $D_{\varphi, u}^{\beta}: H(p, q, \phi) \rightarrow H_{\mu, 0}^{\infty}$ follows. 
As consequences of the above theorems we have the following important corollary.

Corollary 8. For $\beta=1,0<p \leq \infty, 1 \leq q \leq \infty, u \in H(\mathbb{D})$, $\phi$ and $\mu$ are normal, and $\varphi$ is an analytic self-map of $\mathbb{D}$.

(a) Then $D_{\varphi, u}^{1}: H(p, q, \phi) \rightarrow H_{\mu}^{\infty}$ is bounded if and only if

$$
M=\sup _{z \in \mathbb{D}} \frac{\mu(z)|u(z)|}{\phi(|\varphi(z)|)\left(1-|\varphi(z)|^{2}\right)^{(1 / q)+1}}<\infty .
$$

(b) When $u=1$, one gets that $D_{\varphi, 1}^{1}: H(p, q, \phi) \rightarrow H_{\mu}^{\infty}$ is bounded if and only if

$$
M=\sup _{z \in \mathbb{D}} \frac{\mu(z)}{\phi(|\varphi(z)|)\left(1-|\varphi(z)|^{2}\right)^{(1 / q)+1}}<\infty .
$$

(c) For $\varphi(z)=z$, then $D_{z, u}^{1}: H(p, q, \phi) \rightarrow H_{\mu}^{\infty}$ is bounded if and only if

$$
M=\sup _{z \in \mathbb{D}} \frac{\mu(z)|u(z)|}{\phi(|z|)\left(1-|z|^{2}\right)^{(1 / q)+1}}<\infty .
$$

(d) For $u=\varphi^{\prime}, D_{\varphi, \varphi^{\prime}}^{1}: H(p, q, \phi) \rightarrow H_{\mu}^{\infty}$ is bounded if and only if

$$
M=\sup _{z \in \mathbb{D}} \frac{\mu(z)\left|\varphi^{\prime}(z)\right|}{\phi(|\varphi(z)|)\left(1-|\varphi(z)|^{2}\right)^{(1 / q)+1}}<\infty .
$$

A simple calculation shows that $D_{\varphi, 1}^{1}-D_{\varphi, 1}^{0}=\varphi C_{\varphi} D$, $D_{z, u}^{1}-D_{z, u}^{0}=z M_{u} D$, and $D_{\varphi, \varphi^{\prime}}^{1}-D_{\varphi, \varphi^{\prime}}^{0}=\varphi D C_{\varphi}$. Thus the corollaries of Stević's paper [22] on boundedness of these operators follow easily.

\section{Compactness of $D_{\varphi, u}^{\beta}$}

In this section we will characterize when weighted composition operators $D_{\varphi, u}^{\beta}$ acting between mixed-norm spaces and weighted-type space are compact. Before stating the results, we show the following lemma whose proof can be obtained by adapting the proof of Lemma 4 of [25].

Lemma 9. Suppose $\beta \geq 0,0<p \leq \infty, 1 \leq q \leq \infty$, $\phi$ and $\mu$ are normal, and $\varphi$ is a holomorphic self-map of $\mathbb{D}$. Then the operator $D_{\varphi, u}^{\beta}: H(p, q, \phi) \rightarrow H_{\mu}^{\infty}$ is compact if and only if $D_{\varphi, u}^{\beta}: H(p, q, \phi) \rightarrow H_{\mu}^{\infty}$ is bounded and for any bounded sequence $\left\{f_{k}\right\}_{k \in \mathbb{N}}$ in $H(p, q, \phi)$ which converges to zero uniformly on compact subsets of $D$, we have

$$
\left\|D_{\varphi, u}^{\beta} f_{k}\right\|_{H_{\mu}^{\infty}} \longrightarrow 0 \text {, }
$$

as $k \rightarrow \infty$.
Theorem 10. Suppose $\beta \geq 0,0<p \leq \infty, 1 \leq q \leq \infty, \phi$ and $\mu$ are normal, and $\varphi$ is a holomorphic self-map of $\mathbb{D}$. Then the operator $D_{\varphi, u}^{\beta}: H(p, q, \phi) \rightarrow H_{\mu}^{\infty}$ is compact if and only if $D_{\varphi, u}^{\beta}: H(p, q, \phi) \rightarrow H_{\mu}^{\infty}$ is bounded and

$$
\lim _{|\varphi(z)| \rightarrow 1} \frac{\mu(z)|u(z)|}{\phi(|\varphi(z)|)\left(1-|\varphi(z)|^{2}\right)^{(1 / q)+\beta}}=0 .
$$

Proof. Suppose, $D_{\varphi, u}^{\beta}: H(p, q, \phi) \rightarrow H_{\mu}^{\infty}$ is compact. Then it is bounded. Suppose (67) is not true. Then there is a sequence $\left\{z_{k}\right\}_{k \in \mathbb{N}}$ such that $\varphi\left(z_{k}\right) \rightarrow 1$ as $z_{k} \rightarrow \infty$ and $\delta>0$ such that

$$
\frac{\mu\left(z_{k}\right)\left|u\left(z_{k}\right)\right|}{\phi\left(\left|\varphi\left(z_{k}\right)\right|\right)\left(1-\left|\varphi\left(z_{k}\right)\right|^{2}\right)^{(1 / q)+\beta}} \geq \delta, \quad k \in \mathbb{N} .
$$

Let $g_{k}(z)=f_{\varphi\left(z_{k}\right)}, k \in \mathbb{N}$.

It is obvious that

$$
\left|g_{k}(z)\right|=\left|f_{\varphi\left(z_{k}\right)}\right| \leq C \frac{\left(1-\left|\varphi\left(z_{k}\right)\right|^{2}\right)^{t+1}}{\phi\left(\left|\varphi\left(z_{k}\right)\right|\right)\left(1-\overline{\varphi\left(z_{k}\right)} z\right)^{(1 / q)+t+1}} .
$$

As $k \rightarrow \infty$ we have $\varphi\left(z_{k}\right) \rightarrow 1$. From the above inequality we have $g_{k} \rightarrow 0$ as $k \rightarrow \infty$ uniformly on compact subsets of $\mathbb{D}$. Therefore,

$$
\lim _{k \rightarrow \infty}\left\|D_{\varphi, u}^{\beta} g_{k}\right\|_{H_{\mu}^{\infty}}=0
$$

But from our assumption we have

$$
\begin{aligned}
\left\|D_{\varphi, u}^{\beta} g_{k}\right\|_{H_{\mu}^{\infty}} & =\sup _{z \in \mathbb{D}} \mu(z)|u(z)|\left|g_{k}^{[\beta]}(\varphi(z))\right| \\
& \geq \mu\left(z_{k}\right)\left|u\left(z_{k}\right)\right|\left|g_{k}^{[\beta]}\left(\varphi\left(z_{k}\right)\right)\right| \\
& \geq C \frac{\mu\left(z_{k}\right)\left|u\left(z_{k}\right)\right|}{\phi\left(\left|\varphi\left(z_{k}\right)\right|\right)\left(1-\left|\varphi\left(z_{k}\right)\right|^{2}\right)^{(1 / q)+\beta}} \\
& \geq C \delta>0,
\end{aligned}
$$

when $k \rightarrow \infty$, which is a contradiction.

Suppose $D_{\varphi, u}^{\beta}: H(p, q, \phi) \rightarrow H_{\mu}^{\infty}$ is bounded and (67) holds. Then for every $\varepsilon>0$ there exist $\rho \in(0,1)$ such that, when $\rho<|\varphi(z)|<1$,

$$
\frac{\mu(z)|u(z)|}{\phi(|\varphi(z)|)\left(1-|\varphi(z)|^{2}\right)^{(1 / q)+\beta}}<\varepsilon .
$$

Consider $h(z)=1 / \Gamma(1+\beta)$. Obviously, $h(z) \in H(p, q, \phi)$ :

$$
\begin{aligned}
\|u\|_{H_{\mu}^{\infty}} & =\left\|D_{\varphi, u}^{\beta} h\right\|_{H_{\mu}^{\infty}} \\
& \leq\left\|D_{\varphi, u}^{\beta}\right\|_{H(p, q, \phi) \rightarrow H_{\mu}^{\infty}}\|h\|_{H(p, q, \phi)}
\end{aligned}
$$


Assume that $\left\{h_{k}\right\}_{k \in \mathbb{N}}$ is bounded sequence in $H(p, q, \phi)$, that is, $\sup _{k}\left\|h_{k}\right\|_{H(p, q, \phi)}<L$, and converges to zero uniformly on compact subsets of $\mathbb{D}$ as $k \rightarrow \infty$. For $\rho<|\varphi(z)|<1$,

$$
\begin{aligned}
\mu(z)|u(z)|\left|h_{k}^{[\beta]}(\varphi(z))\right| \leq & C \frac{\mu(z)|u(z)|}{\phi(\varphi(z))\left(1-|\varphi(z)|^{2}\right)^{(1 / q)+\beta}} \\
& \times\|h\|_{H(p, q, \phi)}<L C \varepsilon .
\end{aligned}
$$

Now, consider the case $|\varphi(z)| \leq \rho$. We have

$$
\begin{gathered}
h_{k}^{[\beta]}(\varphi(z))=\frac{\Gamma(1+\beta)}{2 \pi i} \int_{|w|=\xi} h_{k}(w) F\left(1,1+\beta ; 1 ; \frac{\varphi(z)}{w}\right) \\
\cdot \frac{1}{w} d w, \quad|\varphi(z)|<|w| .
\end{gathered}
$$

Putting $w=\xi e^{i \psi}$ gives us

$$
\begin{aligned}
& h_{k}^{[\beta]}(\varphi(z))=\frac{\Gamma(1+\beta)}{2 \pi} \\
& \times \int_{0}^{2 \pi} h_{k}(w) F\left(1,1+\beta ; 1 ; \frac{\varphi(z)}{w}\right) d \psi \\
& =\frac{\Gamma(1+\beta)}{2 \pi} \\
& \times \int_{0}^{2 \pi} h_{k}(w) \frac{1}{(1-(\varphi(z) / w))^{1+\beta}} d \psi, \\
& \left|h_{k}^{[\beta]}(\varphi(z))\right| \leq \frac{\Gamma(1+\beta)}{2 \pi} \\
& \times \int_{0}^{2 \pi}\left|h_{k}(w)\right| \frac{1}{|1-(\varphi(z) / w)|^{1+\beta}} d \psi \\
& \leq \frac{\Gamma(1+\beta)}{2 \pi} \\
& \times \int_{0}^{2 \pi}\left|h_{k}(w)\right| \frac{1}{(1-(|\varphi(z)| /|w|))^{1+\beta}} d \psi \\
& \leq \frac{\Gamma(1+\beta)}{2 \pi} \int_{0}^{2 \pi}\left|h_{k}(w)\right| \frac{1}{(1-(r / \xi))^{1+\beta}} d \psi \\
& =\frac{\Gamma(1+\beta)}{2 \pi(1-(r / \xi))^{1+\beta}} \int_{0}^{2 \pi}\left|h_{k}(w)\right| d \psi \\
& =\frac{\xi \Gamma(1+\beta)}{2 \pi(\xi-r)^{1+\beta}} \int_{0}^{2 \pi}\left|h_{k}(w)\right| d \psi \\
& \leq \frac{\Gamma(1+\beta)}{2 \pi(\xi-r)^{1+\beta}} \int_{0}^{2 \pi}\left|h_{k}(w)\right| d \psi,
\end{aligned}
$$

where $|\varphi(z)|=r$. Therefore,

$$
\left|h_{k}^{[\beta]}(\varphi(z))\right| \leq C \int_{0}^{2 \pi}\left|h_{k}(w)\right| d \psi
$$

in compact subsets of $\mathbb{D}$. Therefore we have

$$
\begin{aligned}
\mu(z)|u(z)|\left|h_{k}^{[\beta]}(\varphi(z))\right| & \leq\|u\|_{H_{\mu}^{\infty}}\left|h_{k}^{[\beta]}(\varphi(z))\right| \\
& \leq\left|h_{k}^{[\beta]}(\varphi(z))\right| \\
& \leq C \int_{0}^{2 \pi}\left|h_{k}(w)\right| d \psi
\end{aligned}
$$

Since, on compact subsets of $\mathbb{D}, h_{k} \rightarrow 0$ uniformly as $k \rightarrow$ $\infty$, therefore

$$
\begin{aligned}
& \mu(z)|u(z)|\left|h_{k}^{[\beta]}(\varphi(z))\right| \\
& \leq C \int_{0}^{2 \pi}\left|h_{k}(w)\right| d \psi \longrightarrow 0 \quad \text { as } k \longrightarrow \infty
\end{aligned}
$$

From (72) and (73) it follows that $\left\|D_{\varphi, u}^{\beta} h_{k}\right\|_{H_{\mu}^{\infty}} \rightarrow 0$ as $k \rightarrow \infty$. Therefore the operator $D_{\varphi, u}^{\beta}: H(p, q, \phi) \rightarrow H_{\mu}^{\infty}$ is compact.

The following result is obvious as can be seen from the above theorem.

Theorem 11. Suppose $\beta \geq 0,0<p \leq \infty, 1 \leq q \leq \infty, \phi$ and $\mu$ are normal, and $\varphi$ is a holomorphic self-map of $\mathbb{D}$. Then the operator $D_{\varphi, u}^{\beta}: H(p, q, \phi) \rightarrow H^{\infty}$ is compact if and only if $D_{\varphi, u}^{\beta}: H(p, q, \phi) \rightarrow H^{\infty}$ is bounded and

$$
\lim _{|\varphi(z)| \rightarrow 1} \frac{|u(z)|}{\phi(|\varphi(z)|)\left(1-|\varphi(z)|^{2}\right)^{(1 / q)+\beta}}=0 .
$$

\section{Conflict of Interests}

The authors declare that there is no conflict of interests regarding the publication of this paper.

\section{Acknowledgment}

The first author would like to acknowledge the financial support as Junior Research Fellow and the second author as Principal Investigator of the Project NBHM (National Board for Higher Mathematics, India) (2/48(9)/2012/NBHM (R.P.)/RD-II/2936).

\section{References}

[1] G. E. Andrews, R. Askey, and R. Roy, Special Functions, Cambridge University Press, New York, NY, USA, 1999.

[2] N. M. Temme, Special Functions: An Introduction to the Classical Functions of Mathematical Physics, John Wiley \& Sons, New York, NY, USA, 1996. 
[3] G. H. Hardy and J. E. Littlewood, "Some properties of fractional integrals. II," Mathematische Zeitschrift, vol. 34, no. 1, pp. 403439, 1932.

[4] P. L. Duren, Theory of $H^{p}$ Spaces, Academic Press, New York, NY, USA, 1981.

[5] S. Stević, "Norm of weighted composition operators from Bloch space to $H_{\mu}^{\infty}$ on the unit ball," Ars Combinatoria, vol. 88, pp. 125-127, 2008.

[6] D. Clahane and S. Stević, "Norm equivalence and composition operators between Bloch/Lipschitz spaces of the ball," Journal of Inequalities and Applications, vol. 2006, Article ID 61018, 11 pages, 2006.

[7] S. Li and S. Stević, "Weighted composition operators from Bergman-type spaces into Bloch spaces," Proceedings of the Indian Academy of Sciences, vol. 117, no. 3, pp. 371-385, 2007.

[8] S. Li and S. Stević, "Weighted composition operators from $\alpha$ Bloch space to $H^{\infty}$ on the polydisck," Numerical Functional Analysis and Optimization, vol. 28, no. 7-8, pp. 911-925, 2007.

[9] S. Li and S. Stević, "Weighted composition operators from $H^{\infty}$ to Bloch space on the polydisk," Abstract and Applied Analysis, vol. 2007, Article ID 48478, 13 pages, 2007.

[10] S. Li and S. Stević, "Weighted composition operators between $H^{\infty}$ and $\alpha$-Bloch spaces in the unit ball," Taiwanese Journal of Mathematics, vol. 12, no. 7, pp. 1625-1639, 2008.

[11] A. Montes-Rodríguez, "Weighted composition operators on weighted Banach space of analytic function," Journal of the London Mathematical Society, vol. 61, no. 3, pp. 872-884, 2000.

[12] S. Ohno, "Weighted composition operators between $H^{\infty}$ and the Bloch space," Taiwanese Journal of Mathematics, vol. 5, no. 3, pp. 555-563, 2001.

[13] S. Stević, "Weighted composition operators between mixed norm spaces and $H_{\alpha}^{\infty}$ spaces in the unit ball," Journal of Inequalities and Applications, vol. 2007, Article ID 28629, 9 pages, 2007.

[14] X. Zhu, "Weighted composition operator between $H^{\infty}$ and Bergman type spaces," Communications of the Korean Mathematical Society, vol. 21, no. 4, pp. 719-727, 2006.

[15] R. A. Hibschweiler and N. Portnoy, "Composition followed by differentiation between Bergman and Hardy spaces," Rocky Mountain Journal of Mathematics, vol. 35, no. 3, pp. 843-856, 2005.

[16] S. Li and S. Stević, "Generalized composition operators on Zygmand spaces into Bloch type spaces," Journal of Mathematical Analysis and Applications, vol. 338, no. 2, pp. 1282-1295, 2008.

[17] S. Li and S. Stević, "Products of composition and integral type operators from $H^{\infty}$ to the Bloch space," Complex Variables Elliptic Equations, vol. 53, no. 5, pp. 463-474, 2008.

[18] S. Li and S. Stević, "Products of Volterra type operator and composition operator from $H^{\infty}$ and Bloch spaces to Zygmund spaces," Journal of Mathematical Analysis and Applications, vol. 345, no. 1, pp. 40-52, 2008.

[19] S. Ohno, "Products of composition and differentiation between hardy spaces," Bulletin of the Australian Mathematical Society, vol. 73, no. 2, pp. 235-243, 2006.

[20] S. Stević, "Weighted differentiation composition operators from the mixed-norm space to the $n$th weigthed-type space on the unit disk," Abstract and Applied Analysis, vol. 2010, Article ID 246287, 15 pages, 2010.

[21] S. Stević, "Generalized composition operators between mixednorm and some weighted spaces," Numerical Functional Analysis and Optimization, vol. 29, no. 7-8, pp. 959-978, 2008.
[22] S. Stević, "Weighted differentiation composition operators from mixed-norm spaces to weighted-type spaces," Applied Mathematics and Computation, vol. 211, no. 1, pp. 222-233, 2009.

[23] A. L. Shields and D. L. Williams, "Bounded projections, duality and multipliers in spaces of analytic functions," Transactions of the American Mathematical Society, vol. 162, pp. 287-302, 1971.

[24] W. Rudin, Function Theory in the Unit Ball of $\mathbb{C}^{n}$, Springer, New York, NY, USA, 1980.

[25] S. Stević, "Boundedness and compactness of an integral operator in a mixed norm space on the polydisk," Siberian Mathematical Journal, vol. 48, no. 3, pp. 559-569, 2007. 


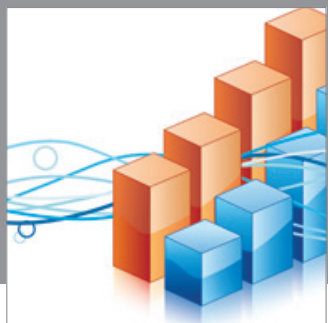

Advances in

Operations Research

mansans

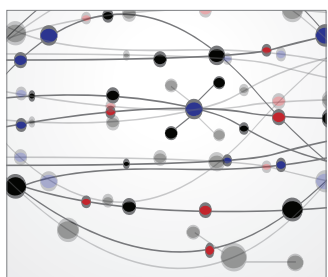

The Scientific World Journal
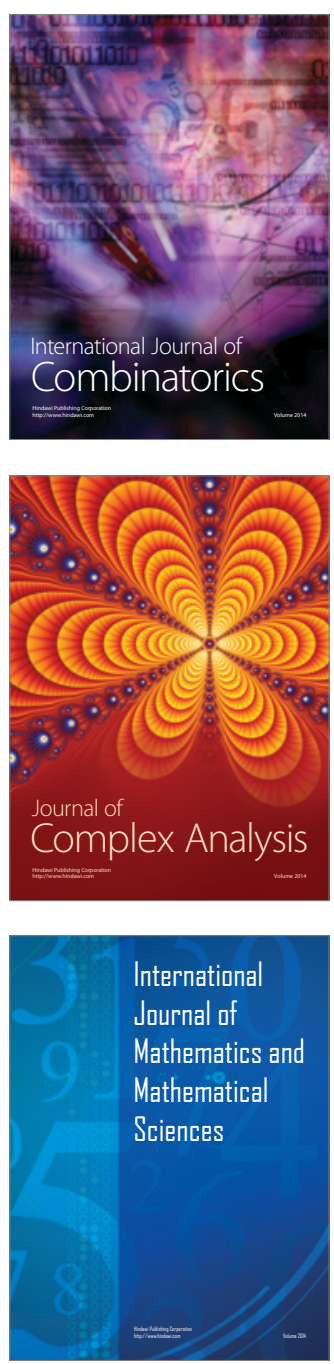
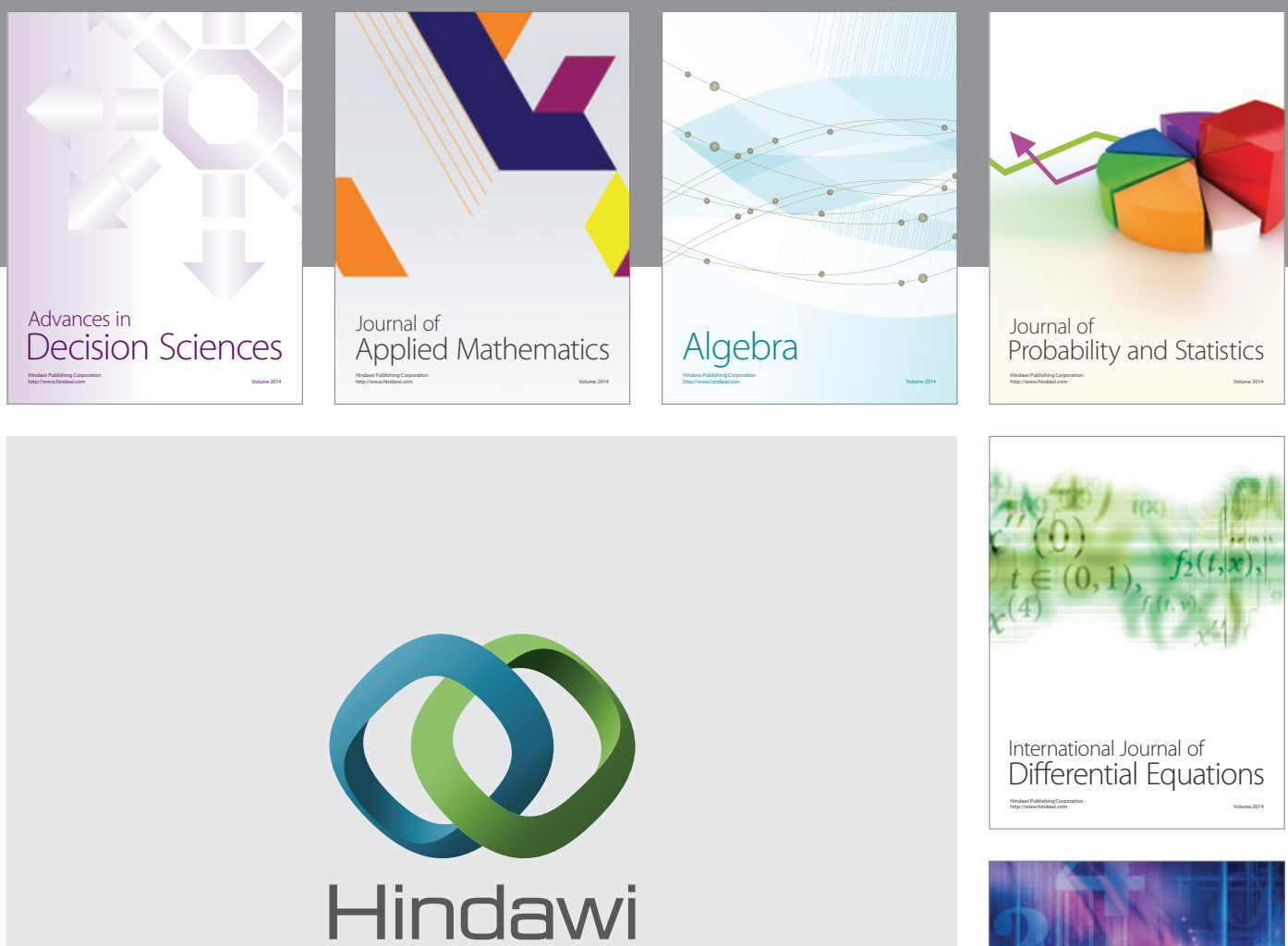

Submit your manuscripts at http://www.hindawi.com
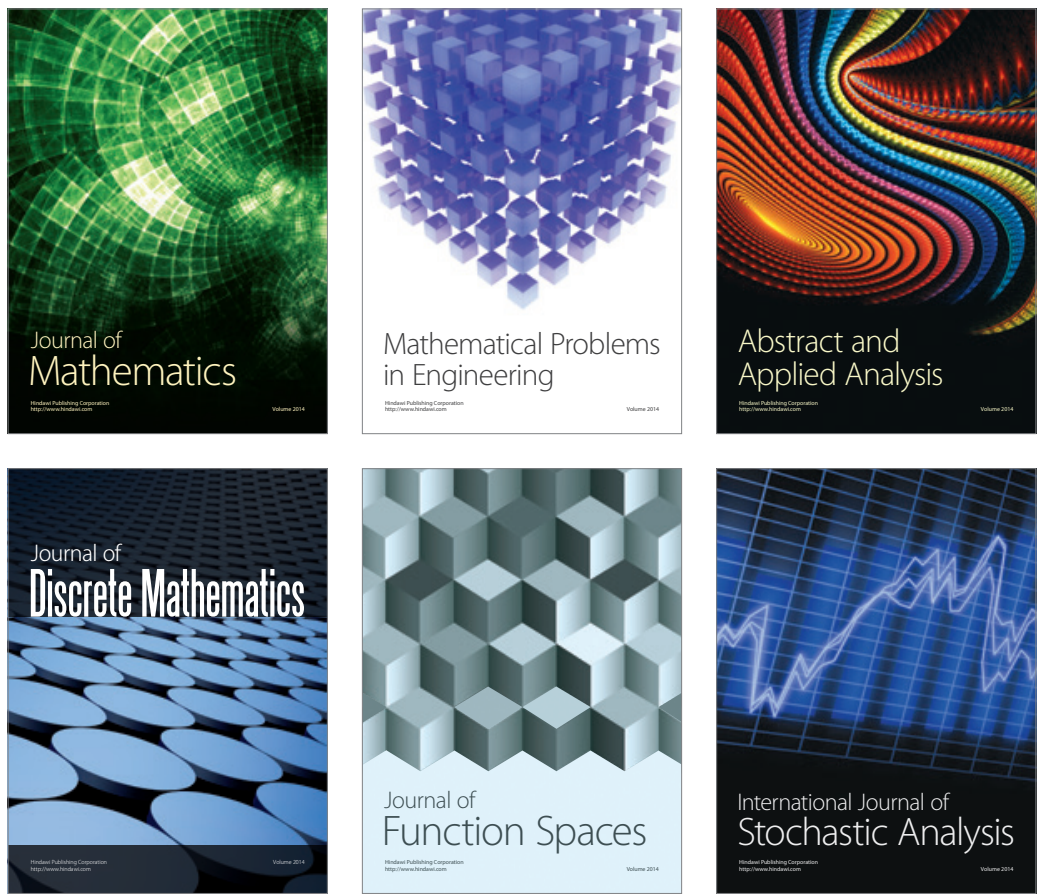

Journal of

Function Spaces

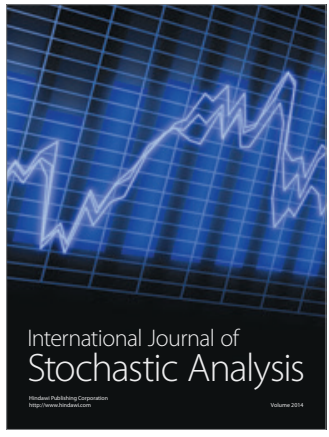

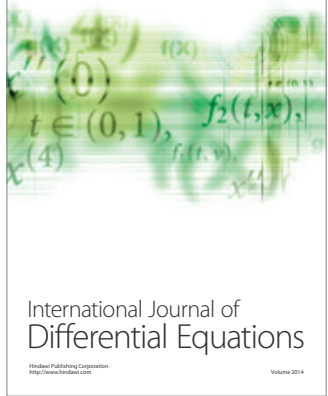
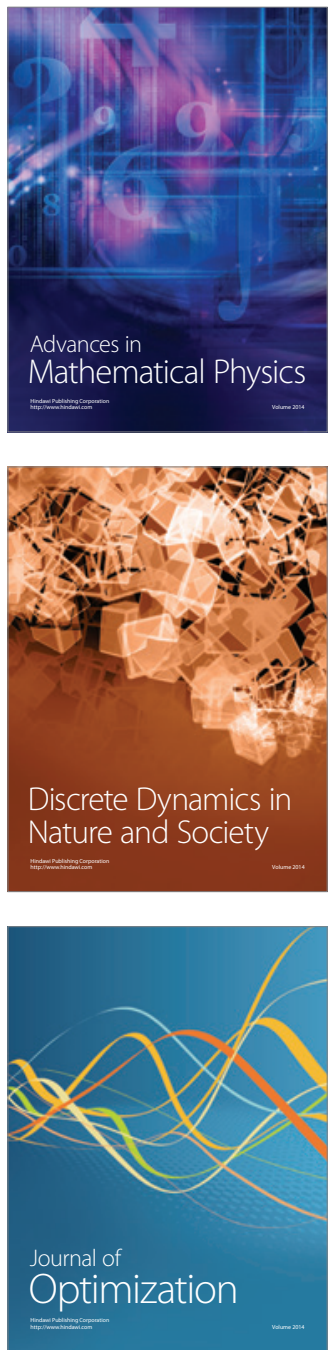